\title{
Analysis and Optimization of Resource Control in High-Speed Railway Wireless Networks
}

\author{
Shengfeng Xu, ${ }^{1,2}$ Gang Zhu, ${ }^{1}$ Chao Shen, ${ }^{1,2}$ Yan Lei, ${ }^{1}$ and Zhangdui Zhong ${ }^{1}$ \\ ${ }^{1}$ State Key Laboratory of Rail Traffic Control and Safety, Beijing Jiaotong University, Beijing 100044, China \\ ${ }^{2}$ The State Key Laboratory of Integrated Services Networks, Xidian University, Xian 710126, China \\ Correspondence should be addressed to Chao Shen; shenchao@bjtu.edu.cn
}

Received 28 November 2013; Revised 21 February 2014; Accepted 26 February 2014; Published 6 April 2014

Academic Editor: Ilse C. Cervantes

Copyright (c) 2014 Shengfeng Xu et al. This is an open access article distributed under the Creative Commons Attribution License, which permits unrestricted use, distribution, and reproduction in any medium, provided the original work is properly cited.

\begin{abstract}
This paper considers a joint optimal design of admission control and resource allocation for multimedia services delivery in highspeed railway (HSR) wireless networks. A stochastic network optimization problem is formulated which aims at maximizing the system utility while stabilizing all transmission queues under the average power constraint. By introducing virtual queues, the original problem is equivalently transformed into a queue stability problem, which can be naturally decomposed into three separate subproblems: utility maximization, admission control, and resource allocation. A threshold-based admission control strategy is proposed for the admission control subproblem. And a distributed resource allocation scheme is developed for the mixed-integer resource allocation subproblem with guaranteed global optimality. Then a dynamic admission control and resource allocation algorithm is proposed, which is suitable for distributed implementation. Finally, the performance of the proposed algorithm is evaluated by theoretical analysis and numerical simulations under realistic conditions of HSR wireless networks.
\end{abstract}

\section{Introduction}

With the rapid development of high-speed railway (HSR) around the world, the wireless communication in HSR networks plays an important role in the recent years [1]. On the one hand, more and more data related with the railway controlling information needs to be transmitted between the train and the ground such that the safety can be guaranteed and the transportation efficiency can be significantly improved. On the other hand, the passengers in the train have an increasingly high demand on multimedia services. However, these requirements on high throughput impose a great challenge over the HSR communication designs due to the fast-varying channel, train penetration loss, and so forth.

There have been some recent works to improve the throughput in HSR wireless networks. A two-hop HSR network architecture was proposed in [2] to provide high datarate services. A HSR communication system based on radio over fiber technology was proposed in [3], which can increase the system throughput and help to reduce the number of handoffs. Multi-input multi-output (MIMO) antennas were employed to improve the throughput performance of the HSR wireless networks $[4,5]$. However, these works were carried out only to improve the throughput performance in HSR wireless networks. Since the buffering is involved at network devices, for example, content servers, it is necessary to consider not only the throughput performance but also the queue stability in HSR wireless networks.

Admission control and resource allocation, as critical parts of radio resource management, play important roles in improving the throughput and ensuring queue stability. In the literature, the energy constrained control algorithm was proposed in [6] to stabilize the queue and maximize the throughput by Lyapunov optimization theory. Paper [7] studied the joint scheduling and admission control problem in a single user scenario and an online learning algorithm was proposed based on the Markov decision process approach and stochastic control theory. However, these existing schemes designed for general communication systems are not easily extended to the scenario considered in this paper, due to the following reasons: (1) in HSR wireless networks, the channel condition cannot remain at the same 
level because of the fast-varying distance between the base station and the train, which causes that the power control along the time has a large influence on system transmission performance [8]; (2) many types of services with different quality-of-service (QoS) requirements and priorities should be supported [9], which makes the admission control and resource allocation for multiple services more challenging.

In HSR wireless networks, few studies have been conducted on admission control and resource allocation. A scheduling and resource allocation mechanism was presented in [10] to maximize the service rate in HSR networks with a cell array architecture. In [11], a multidimensional resource allocation strategy was proposed in downlink orthogonal frequency-division multiplexing (OFDM) system for HSR communications. The optimal resource allocation problem in a cellular/infostation integrated HSR network was investigated in [12], which considered the intermittent network connectivity and multiservice demands. In a relay-assisted HSR network, [13] studied delay-aware fair downlink service scheduling problem with heterogeneous packet arrivals and delay requirements for the services. Paper [14] proposed an effective admission control scheme considering different service priorities for HSR communications with MIMO antennas. However, to the best of our knowledge, the joint admission control and resource allocation problem under the average power constraint in HSR wireless networks is still an open problem.

The main contribution of this paper is a stochastic optimization framework for transmitting multimedia services in HSR wireless networks, which focuses on the joint admission control and resource allocation problem under the average power constraint. Firstly, the joint admission control and resource allocation problem is formulated as a stochastic optimization problem, and then the problem is transformed into a queue stability problem with the help of virtual queues. By the drift-plus-penalty approach [15], the transformed problem can be decomposed into three separate subproblems: utility maximization, admission control, and resource allocation. The former two subproblems are easy to handle and the distributed solutions can be obtained directly, while the mixed-integer resource allocation subproblem is transformed into a single variable problem and a distributed packet loading scheme is developed with guaranteed global optimality. We further propose a dynamic admission control and resource allocation algorithm, which is suitable for distributed implementation in HSR wireless networks. Finally, we present the analysis of algorithm performance by theoretical derivations and simulations under realistic conditions for HSR wireless networks.

1.1. Relation to Prior Work. The Lyapunov drift theory has a long history in the field of discrete stochastic processes and Markov chains [16]. It can be used to directly analyze the characteristics of the control policies in the stochastic stability sense and plays important roles in the dynamic control strategies in queuing networks [17]. Stabilizing queuing networks by minimizing Lyapunov drift was pioneered by Tassiulas and Ephremides in [18]. The Lyapunov drift theory was then extended to the Lyapunov optimization theory [6], which enables optimization of time averages of general network utilities subject to queue stability. A general framework for solving the stochastic network optimization problem based on Lyapunov optimization theory was developed in [15]. This framework has been extended to minimizing a drift-pluspenalty expression in $[6,7,17,19,20]$ for joint queue stability and time average utility optimization. For the engineering applications of Lyapunov optimization theory, interested readers are referred to the aforementioned references for the details.

Our approach in the present paper treats the joint admission control and resource allocation problem associated with average power constraint using Lyapunov drift and Lyapunov optimization theory from [15]. This is the first time, to the best of our knowledge, that the Lyapunov optimization theory is extended into the HSR wireless networks. Considering the features of HSR wireless networks, the Lyapunov optimization theory is successfully applied for solving the joint admission control and resource allocation problem in HSR wireless network.

1.2. Outline of Paper. The rest of the paper is organized as follows. Section 2 describes the system model. The problem formulation and transformation are provided in Section 3. A distributed dynamic admission control and resource allocation algorithm is proposed in Section 4. Some numerical results and discussions are shown in Section 5. Finally, conclusions are drawn in Section 6.

Notations. In this paper, $\mathbb{E}[\cdot]$ denotes expectation. $\lfloor x\rfloor=\max$ $\{n \in \mathbb{Z} \mid n \leq x\}$. $\max [x, y]$ and $\min [x, y]$ mean the maximum and minimum between $x$ and $y$, respectively.

\section{System Model}

In this paper, a two-hop HSR wireless network architecture is considered, as shown in Figure 1, which consists of a backbone network, $K$ content servers (CSs), several base stations (BSs), a relay station (RS), and some users in the train. The BSs deployed along the rail line can provide continuous data packets delivery. The distributed CSs connected to the BSs via wireline links are deployed in the backbone network to offload the data traffic [21]. The RS with powerful antennas installed on the top of the train is used for communicating with the BSs so that the large train penetration loss can be well resolved. The RS is further connected to the access points (APs) which can be accessed by the users inside the train. Thus, there is a two-hop wireless link, consisting of the BS-RS link and the AP-Users link. If the users on the train request multimedia services during a trip, the data packets of the requested services are then delivered from the corresponding CS to the RS via a BS. Suppose that the data transmission rate in the AP-Users link is sufficiently large; hence the data packet can be successfully received if it has been delivered to the RS.

2.1. Time-Distance Mapping. Consider a train traveling from an origin station to a destination station within the time 


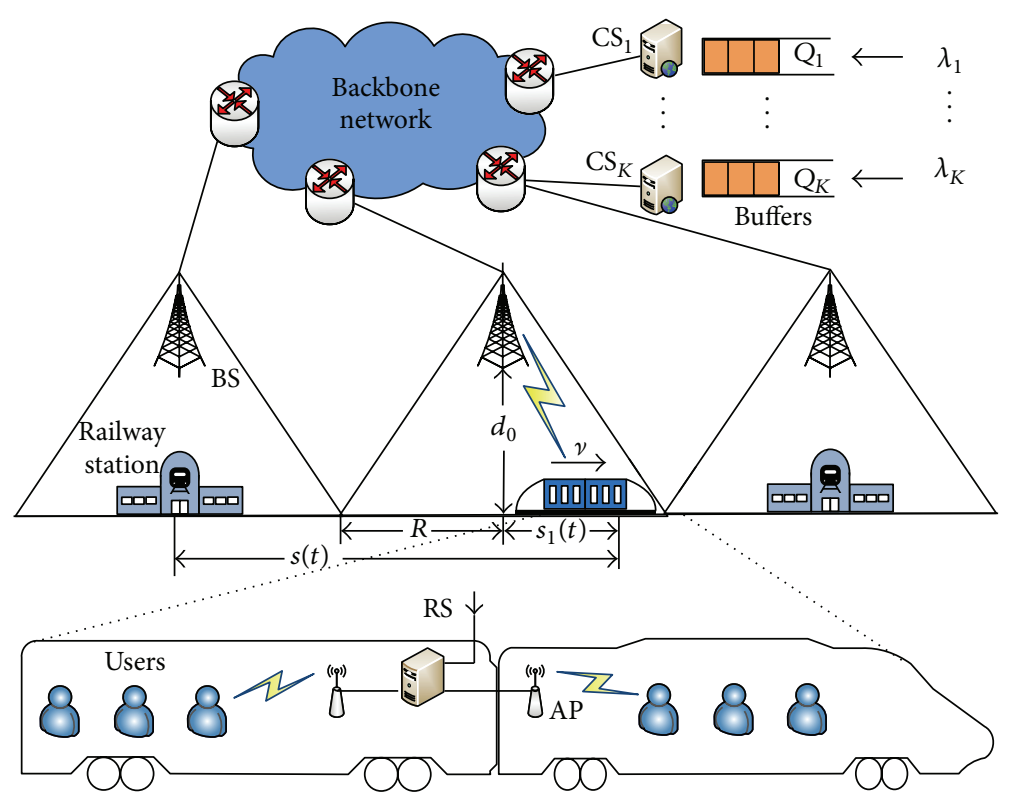

FIGURE 1: System model.

duration $[0, T]$. The whole time is divided into slots of equal duration $T_{s}$. Without loss of generality, we assume that the train starts at the centre of the first cell and the train moving speed during the slot $t$ keeps constant, denoted by $v(t)$; thus the traveled distance until slot $t$ is given by $s(t)=\sum_{\tau=0}^{t} v(\tau) T_{s}$. The train location between two adjacent BSs at slot $t$ is $s_{1}(t)=s(t) \bmod 2 R$, where $R$ is the cell radius. Define a timedistance mapping function $d(t)$ as the distance between BS and RS at slot $t$; that is, $d(t):[0, T] \rightarrow\left[d_{0}, d_{\max }\right]$, where $d_{\max }=\sqrt{R^{2}+d_{0}^{2}}$ and $d_{0}$ is the distance between each BS and the rail line as shown in Figure 1. The mapping function $d(t)$ can be expressed by

$$
d(t)= \begin{cases}\sqrt{s_{1}(t)^{2}+d_{0}^{2}}, & \text { if } 0 \leq s_{1}(t)<R \\ \sqrt{\left(2 R-s_{1}(t)\right)^{2}+d_{0}^{2}}, & \text { if } R \leq s_{1}(t)<2 R\end{cases}
$$

Here we assume that the distance $d(t)$ does not change within slot $t$ since $T_{s}$ is very small.

2.2. Physical Layer Model. For HSR wireless networks, the channel condition cannot remain at the same level due to the fast-varying distance between BS and RS. Only the lineof-sight (LOS) path in the BS-RS link is available at most of the time, which was confirmed by engineering measurements $[22,23]$. The service provided by the independent identical distributed (i.i.d.) fading channels is a deterministic timelinear function, just like the AWGN channel [24]. Therefore, the wireless channel in the BS-RS link can be assumed to be an additive white Gaussian noise channel (AWGN) with LOS path loss [8]. At the same time, the power control along the travel time in HSR wireless networks is important. Denote by $P(t)$ the transmit power of the BS at slot $t$, which is limited by the maximum value $P_{\max }$ and average value $P_{\mathrm{av}}$. With the help of mapping function $d(t)$ and according to Shannon's theorem [25], the transmission rate of the wireless channel between $\mathrm{BS}$ and RS at slot $t$ can be expressed by

$$
R(t)=W \log _{2}\left(1+\frac{P(t)}{N(t)}\right) \text { bits/s, }
$$

where $N(t)=W N_{0} d^{\alpha}(t), W$ is the system bandwidth, $N_{0}$ is the noise power spectral density, and $\alpha$ is the path loss exponent. Suppose that the packets have equal size of $L$ bits; hence the link capacity $C(t)$ at slot $t$ can be denoted as the maximum number of packets; that is, $C(t)=\left\lfloor R(t) T_{s} / L\right\rfloor$. Note that the maximum capacity $C_{\max }$ can be obtained when $d(t)=d_{0}$ and $P(t)=P_{\max }$.

2.3. Service Model. Assume that there are $K$ types of services in the HSR wireless networks and the service type set is denoted by $\mathscr{K} \triangleq\{1, \ldots, K\}$. We further assume that $\mathrm{CS}_{k}$ is equipped with a buffer and can provide service $k$, for $k \in \mathscr{K}$. Let $\mathbf{A}(t)=\left[A_{1}(t), \ldots, A_{K}(t)\right]^{T}$ represent the packet arrival vector, where $A_{k}(t)$ denotes the number of new arrival packets of service $k$ at slot $t$. The packet arrival process for each service is assumed to be i.i.d. across slots. Suppose that, in general, $A_{k}(t)$ follows a truncated Poisson distribution $f_{k}(b)$ with average arrival rate $\lambda_{k}=\mathbb{E}\left[A_{k}(t)\right]$ for service $k$, and the distribution $f_{k}(b)$ can be written as

$$
f_{k}(b)=\exp \left(-\lambda_{k}\right) \frac{\lambda_{k}^{b}}{b !}, \quad b=0, \ldots, B_{k}
$$

where $B_{k}$ denotes the maximum number of arrival packets per slot for service $k$ and can be found assuming $f_{k}\left(B_{k}\right) \rightarrow 0$.

Let $\mathbf{Q}(t)=\left[Q_{1}(t), \ldots, Q_{K}(t)\right]^{T}$ represent the vector of current queue backlogs, where $Q_{k}(t)$ denotes the number of packets at the beginning of slot $t$ in the buffer of $\mathrm{CS}_{k}$. The dynamics of each buffer are controlled by admission 
control (AC) and resource allocation (RA) actions. Specifically, at each slot, the AC action determines the number of packets from the newly arriving packets to be stored into the buffer. And the RA action determines the number of packets removed from the buffer for transmission. Let $r_{k}(t) \epsilon$ $\left[0, A_{k}(t)\right]$ and $\mu_{k}(t) \in\left[0, Q_{k}(t)\right]$ denote the AC action and RA action for service $k$ at slot $t$, respectively. Thus, the queue dynamics can be characterized by

$$
Q_{k}(t+1)=Q_{k}(t)-\mu_{k}(t)+r_{k}(t), \quad \forall k \in \mathscr{K}
$$

Notice that for any slot $t$, without AC actions, $r_{k}(t)=A_{k}(t)$. Here we assume that the arrival packets at slot $t$ can only be transmitted at slot $t+1$.

\section{Problem Formulation and Transformation}

3.1. Problem Formulation. In this paper, the objective of the joint AC and RA problem is to maximize a sum of utility functions under time average constraints by designing a dynamic algorithm over a trip of the train. We define that $\phi_{k}\left(r_{k}\right)$ is a utility function to present throughput benefit for service $k$, which is nondecreasing concave continuous with $r_{k}$. Throughout this work, the following notation for the longterm time average expectation of any quantity $z$ is defined:

$$
\bar{z}:=\lim _{t \rightarrow \infty} \frac{1}{t} \sum_{\tau=0}^{t-1} \mathbb{E}[z(\tau)] .
$$

In particular, $\bar{Q}_{k}$ represents the average queue backlog in the buffer of $\mathrm{CS}_{k}$ and $\bar{P}$ represents the average power consumption along the travel time. Here we introduce the definitions of queue stability as follows [15].

Definition 1. A single queue $Q(t)$ is mean rate stable if $\lim _{t \rightarrow \infty}(\mathbb{E}[Q(t)] / t)=0$.

Definition 2. A single queue $Q(t)$ is strongly stable if $\bar{Q}<\infty$.

From Definition 2, a queue is strongly stable if it has a bounded time average backlog. Strong stability implies mean rate stability according to [15]. Throughout this paper, we use the term "stability" to refer to strong stability. Define $\Omega(t) \triangleq$ $(\mathbf{A}(t), C(t))$ as the observed network event at slot $t$. For each slot $t$, observing the event $\Omega(t)$ and the queue state $\mathbf{Q}(t)$, the AC actions $r_{k}(t)$ and RA actions $\mu_{k}(t)$ should be made for $k \in \mathscr{K}$. The joint AC and RA problem is formulated as

$$
\begin{array}{ll}
\text { (P1) maximize } & \sum_{k \in \mathscr{K}} \phi_{k}\left(\bar{r}_{k}\right) \\
\text { subject to } & \bar{P} \leq P_{\mathrm{av}}, \quad P(t) \leq P_{\max }, \quad \forall t \in[0, T] \\
& \bar{Q}_{k}<\infty, \quad \forall k \in \mathscr{K},
\end{array}
$$

$$
\begin{aligned}
& 0 \leq \sum_{k \in \mathscr{K}} \mu_{k}(t) \leq C(t), \quad \forall t \in[0, T], \\
& \mu_{k}(t) \in\left[0, Q_{k}(t)\right], \quad \forall t \in[0, T], \\
& r_{k}(t) \in\left[0, A_{k}(t)\right], \quad \forall t \in[0, T],
\end{aligned}
$$

variables $r_{k}(t), \mu_{k}(t), P(t), \quad \forall k \in \mathscr{K}, \quad t \in[0, T]$,

where (6b) corresponds to the power constraint and (6c) corresponds to the queue stability constraints for all queues. Problem (P1) is a stochastic optimization problem [15], but it cannot be solved efficiently owing to the difficulty from the objective function (6a) and the average power constraint in (6b). In order to better characterize the problem (P1) and develop an efficient algorithm, we consider the problem transformation, which consists of two steps, that is, objective function transformation and average power constraint transformation as presented in the following subsections.

3.2. Objective Function Transformation. Since problem (P1) involves maximizing a function of time averages, it is hard to handle. Based on the dynamic stochastic optimization theory [15], it can be transformed into an equivalent problem that involves maximizing a single time average of a function. This transformation is achieved through the use of auxiliary variables $\gamma_{k}(t)$ and corresponding virtual queues $Z_{k}(t)$ with queue evolutions:

$$
Z_{k}(t+1)=\max \left[Z_{k}(t)-r_{k}(t), 0\right]+\gamma_{k}(t), \quad \forall k \in \mathscr{K},
$$

where the initial condition is assumed that $Z_{k}(0)=0, \forall k \in$ $\mathscr{K}$. Intuitively, the auxiliary variables $\gamma_{k}(t)$ can be viewed as the "arrivals" of virtual queues $Z_{k}(t)$, while $r_{k}(t)$ can be viewed as the service rate of such virtual queues.

Then, we consider the following transformed problem:

$$
\begin{array}{cl}
\text { (P2) maximize } & \sum_{k \in \mathscr{K}} \overline{\phi_{k}\left(\gamma_{k}\right)} \\
\text { subject to } \quad \bar{\gamma}_{k} \leq \bar{r}_{k}, \quad \forall k \in \mathscr{K}, \\
0 \leq \gamma_{k}(t) \leq B_{k}, \quad \forall k \in \mathscr{K}, \quad t \in[0, T], \\
(6 \mathrm{~b})-(6 \mathrm{f}), \\
\text { variables } \gamma_{k}(t), r_{k}(t), \mu_{k}(t), P(t), \\
\forall k \in \mathscr{K}, \quad t \in[0, T] .
\end{array}
$$

Constraint $(8 \mathrm{~b})$ corresponds to the stability of the virtual queue $Z_{k}(t)$, since $\bar{\gamma}_{k}$ and $\bar{r}_{k}$ are regarded as the time-averaged arrival rate and the time-averaged service rate for the virtual queue $Z_{k}(t)$, respectively. Specifically, from (7) we can obtain that $Z_{k}(t+1) \geq Z_{k}(t)-r_{k}(t)+\gamma_{k}(t)$. By summing this inequality over time slots $\tau \in\{0,1, \ldots, t-1\}$ and then dividing the result by $t$, we have that $\left(Z_{k}(t)-Z_{k}(0)\right) / t+$ $(1 / t) \sum_{\tau=0}^{t-1} r_{k}(\tau) \geq(1 / t) \sum_{\tau=0}^{t-1} \gamma_{k}(t)$. With $Z_{k}(0)=0$, taking 
expectations of both sides yields that $\lim _{t \rightarrow \infty}\left(\mathbb{E}\left[Z_{k}(t)\right] / t\right)+$ $\bar{r}_{k} \geq \bar{\gamma}_{k}$. If the virtual queues $Z_{k}(t)$ are mean rate stable, then $\lim _{t \rightarrow \infty}\left(\mathbb{E}\left[Z_{k}(t)\right] / t\right)=0$, so that constraint $(8 \mathrm{~b})$ can be satisfied. Notice that we will prove the strong stability of the virtual queues $Z_{k}(t)$ in Lemma 7 later.

Lemma 3. Problem (P1) and problem (P2) are equivalent.

Proof. The proof of Lemma 3 follows [26] and a sketch of the proof is provided in Appendix A.

3.3. Average Power Constraint Transformation. To handle the average power constraint in (6b), we define a virtual queue $X_{k}(t)$ for each $k \in \mathscr{K}$, which has the following dynamic update equation:

$$
X_{k}(t+1)=\max \left[X_{k}(t)-P_{\mathrm{av}}, 0\right]+P(t),
$$

where $P(t)$ and $P_{\mathrm{av}}$ can be viewed as the "arrivals" and "offered service" at slot $t$, respectively.

Based on [15, Chapter 4], if the virtual queue $X_{k}(t)$ is mean rate stable for $k \in \mathscr{K}$, that is, $\lim _{t \rightarrow \infty}\left(\mathbb{E}\left[X_{k}(t)\right] / t\right)=0$, then the average power constraint $\bar{P} \leq P_{\text {av }}$ can be satisfied. This holds because if the backlog in the virtual queue is stabilized, it must be the case that the time average arrival rate (corresponding to $\bar{P}$ ) is not larger than the service rate (corresponding to $P_{\mathrm{av}}$ ). Therefore, the average power constraint in (6b) can be transformed into a single queue stability problem.

\section{The Distributed Dynamic AC and RA Algorithm}

In this section, the dynamic stochastic optimization approach is applied to solve problem (P2), which seeks to maximize the sum of time-averaged utility functions subject to queue stability constraints. Firstly, the problem (P2) is decomposed into three separate subproblems by the drift-plus-penalty approach. Then a distributed dynamic AC and RA algorithm is proposed. Finally, the performance of the proposed algorithm is analyzed by theoretical derivations.

4.1. Lyapunov Drift. Define $\mathbf{X}(t)$ and $\mathbf{Z}(t)$ as a vector of all virtual queues $X_{k}(t)$ and $Z_{k}(t)$ for $k \in \mathscr{K}$, respectively. We denote by $\Theta(t)$ the combined vector of all virtual queues and all actual queues; namely,

$$
\boldsymbol{\Theta}(t) \triangleq\left[\mathbf{Q}^{T}(t), \mathbf{X}^{T}(t), \mathbf{Z}^{T}(t)\right]^{T}
$$

The quadratic Lyapunov function is defined as [15]

$$
L(\Theta(t)) \triangleq \frac{1}{2} \sum_{k \in \mathscr{K}}\left(Q_{k}(t)^{2}+X_{k}(t)^{2}+Z_{k}(t)^{2}\right) .
$$

Then the one-slot conditional Lyapunov drift $\Delta(\Theta(t))$ at slot $t$ is given by

$$
\Delta(\Theta(t))=\mathbb{E}[L(\Theta(t+1))-L(\Theta(t)) \mid \Theta(t)],
$$

which admits the following lemma.
Lemma 4. Under any $A C$ actions and $R A$ actions at slot $t$, and for any value of $\Theta(t)$, we have

$$
\Delta(\Theta(t)) \leq D+\mathbb{E}[G(t) \mid \Theta(t)],
$$

where $D$ is a finite constant defined by

$$
D \triangleq \frac{1}{2} \sum_{k \in \mathscr{K}}\left[P_{\max }^{2}+P_{a v}^{2}+3 B_{k}^{2}+C_{\max }^{2}\right]
$$

and $G(t)$ is defined by

$$
\begin{aligned}
G(t) \triangleq & \sum_{k \in \mathscr{K}} Q_{k}(t)\left[r_{k}(t)-\mu_{k}(t)\right] \\
& +\sum_{k \in \mathscr{K}} Z_{k}(t)\left(\gamma_{k}(t)-r_{k}(t)\right) \\
& +\sum_{k \in \mathscr{K}} X_{k}(t)\left[P(t)-P_{a v}\right]
\end{aligned}
$$

Proof. The proof of Lemma 4 is provided in Appendix B.

4.2. The Drift-Plus-Penalty Expression. Instead of directly minimizing the upper bound $\mathbb{E}[G(t)]$ by taking AC actions and RA actions, we desire to jointly stabilize all queues and maximize the sum of utility $\sum_{k \in \mathscr{K}} \phi_{k}\left(\gamma_{k}(t)\right)$. The drift-pluspenalty theory in [6] approaches this by greedily minimizing the following "drift-plus-penalty" expression:

$$
\mathbb{E}\left[G(t)-V \sum_{k \in \mathscr{K}} \phi_{k}\left(\gamma_{k}(t)\right)\right]
$$

where $V \geq 0$ is a parameter that represents the weight on how much we emphasize the sum utility maximization.

We observe that the objective function in (16) is of separable structure, which motivates us to determine the auxiliary variables $\gamma_{k}(t)$ and AC actions $r_{k}(t)$ as well as RA actions $\mu_{k}(t)$ in an alternative optimization fashion. The overall minimization problem (16) is decomposed into three separate subproblems. Specifically, isolating the $\gamma_{k}(t)$ variables from (16) gives the following utility maximization subproblem:

$$
\begin{array}{ll}
\max _{\left\{\gamma_{k}(t)\right\}} & \sum_{k \in \mathscr{K}}\left[V \phi_{k}\left(\gamma_{k}(t)\right)-Z_{k}(t) \gamma_{k}(t)\right] \\
\text { s.t. } & 0 \leq \gamma_{k}(t) \leq B_{k}, \quad \forall k \in \mathscr{K}, t \in[0, T] .
\end{array}
$$

Similarly, isolating the AC actions $r_{k}(t)$ from (16) leads to the following admission control subproblem:

$$
\begin{array}{ll}
\max _{\left\{r_{k}(t)\right\}} & \sum_{k \in \mathscr{K}}\left[\left(Z_{k}(t)-Q_{k}(t)\right) r_{k}(t)\right] \\
\text { s.t. } & 0 \leq r_{k}(t) \leq A_{k}(t), \quad \forall k \in \mathscr{K}, t \in[0, T] .
\end{array}
$$


Also, isolating the RA actions $\mu_{k}(t)$ from (16) gives the following resource allocation subproblem:

$$
\begin{array}{ll}
\max _{\left\{\mu_{k}(t)\right\}, P(t)} & \sum_{k \in \mathscr{K}}\left[Q_{k}(t) \mu_{k}(t)-X_{k}(t) P(t)\right] \\
\text { s.t. } \quad & 0 \leq \mu_{k}(t) \leq Q_{k}(t), \\
& \mu_{k}(t) \in \mathbb{N}, \quad \forall k \in \mathscr{K}, \quad t \in[0, T], \\
& \sum_{k \in \mathscr{K}} \mu_{k}(t) \leq C(t), \quad t \in[0, T], \\
& P(t) \leq P_{\max }, \quad t \in[0, T],
\end{array}
$$

where $P(t)$ is related to $\mu_{k}(t)$ since a larger $\mu_{k}(t)$ requires more power consumption. These separate subproblems can be computed in a decentralized fashion, as stated below.

4.3. Utility Maximization. The utility maximization subproblem ((17a) and (17b)) can be decoupled into $K$ separate maximization problems. Specifically, $\mathrm{CS}_{k}$ keeps track of $Z_{k}(t)$ and determines the optimum $\gamma_{k}(t)$ by solving the following problem:

$$
\begin{array}{ll}
\max _{\gamma_{k}(t)} & V \phi_{k}\left(\gamma_{k}(t)\right)-Z_{k}(t) \gamma_{k}(t) \\
\text { s.t. } & 0 \leq \gamma_{k}(t) \leq B_{k}, \quad t \in[0, T]
\end{array}
$$

Notice that the key point to solve (20a) and (20b) is the choice of the utility function, which is contingent on the purpose of the networking application or the prerogative of HSR network designer. For example, in order to represent the maximum desired delivery ratio for each service, the piecewise linear utility function can be considered for service $k$ as follows:

$$
\phi_{k}\left(\gamma_{k}\right)=\nu_{k} \min \left[\gamma_{k}, x_{k} \lambda_{k}\right]
$$

where $v_{k}>0$ and $x_{k}>0$ represent the priority and the maximum desired delivery ratio of service $k$, respectively. In general, $0 \leq x_{k} \leq 1$ and $x_{k} \lambda_{k} \leq B_{k}$ for $k \in \mathscr{K}$. Thus the optimal solution to problem (20a) and (20b) is given by

$$
\gamma_{k}(t)= \begin{cases}x_{k} \lambda_{k}, & \text { if } Z_{k}(t) \leq V \nu_{k}, \\ 0, & \text { otherwise. }\end{cases}
$$

Alternatively, the following strictly concave function can serve as the utility function for service $k$ :

$$
\phi_{k}\left(\gamma_{k}\right)=\ln \left(1+\nu_{k} \gamma_{k}\right)
$$

which can be regarded as an accurate approximation of the proportionally fair utility function if the same $v_{k}$ is selected with a large value for all $k \in \mathscr{K}$. In this case, the optimal solution to problem (20a) and (20b) can be obtained by

$$
\gamma_{k}(t)=\left[\frac{V}{Z_{k}(t)}-\frac{1}{\nu_{k}}\right]_{0}^{B_{k}},
$$

where the operation $[y]_{0}^{a}$ is equal to $y$ if $0<y<a, 0$ if $y \leq 0$, and $a$ if $y \geq a$.
4.4. Admission Control. The admission control subproblem ((18a) and (18b)) can be also decoupled into $K$ separate maximization problems. Specifically, $\mathrm{CS}_{k}$ chooses the AC action $r_{k}(t)$ by solving the following optimization problem:

$$
\begin{array}{ll}
\max _{r_{k}(t)} & \left(Z_{k}(t)-Q_{k}(t)\right) r_{k}(t) \\
\text { s.t. } & 0 \leq r_{k}(t) \leq A_{k}(t), \quad t \in[0, T] .
\end{array}
$$

It is immediate to see that the optimal solution depends on the queue backlog of $Z_{k}(t)$ and $Q_{k}(t)$, which is given by

$$
r_{k}(t)= \begin{cases}A_{k}(t), & \text { if } Z_{k}(t) \geq Q_{k}(t), \\ 0, & \text { otherwise. }\end{cases}
$$

We note that (26) is a simple threshold-based admission control strategy. On the one hand, when the queue backlog $Q_{k}(t)$ is not larger than the threshold $Z_{k}(t)$, then all the newly arriving packets are admitted into the buffer in $\mathrm{CS}_{k}$. Essentially, this not only reduces the value of $Z_{k}(t+1)$ so as to push $\bar{\gamma}_{k}$ closer to $\bar{r}_{k}$, but also increases the average throughput $\bar{r}_{k}$ so as to improve the utility. On the other hand, when the queue backlog $Q_{k}(t)$ is larger than the threshold $Z_{k}(t)$, then all the newly arriving packets will be dropped to ensure the network stability. Finally, we emphasize that the $\mathrm{AC}$ actions for all services are made in a distributed manner with only local queue backlog information and packet arrival information.

4.5. Resource Allocation. The resource allocation subproblem ((19a)-(19d)) at slot $t$ can be explicitly expressed as

$$
\begin{gathered}
\max _{\left\{\mu_{k}(t)\right\}, P(t)} \quad M(t) \triangleq \sum_{k \in \mathscr{K}}\left[Q_{k}(t) \mu_{k}(t)-X_{k}(t) P(t)\right] \\
\text { s.t. } \quad 0 \leq \mu_{k}(t) \leq Q_{k}(t), \quad \mu_{k}(t) \in \mathbb{N}, \quad \forall k \in \mathscr{K}, \\
\sum_{k \in \mathscr{K}} \mu_{k}(t) \leq C(t)=\left\lfloor\frac{T_{s} W \log _{2}(1+P(t) / N(t))}{L}\right\rfloor, \\
P(t) \leq P_{\max } .
\end{gathered}
$$

The problem $(27 a)-(27 d)$ is a mixed-integer programming (MIP) problem, including a continuous variable $P(t)$ and $K$ integer variables $\mu_{k}(t)$, which cannot be solved efficiently [27]. The main difficulty of problem (27a)-(27d) comes from the integer nature of $\mu_{k}(t)$. However, we will show that problem $(27 \mathrm{a})-(27 \mathrm{~d})$ can be transformed into a single variable problem, which is easy to handle. In the sequel of this subsection, we will omit the time index for brevity.

Firstly, as for constraint (27c), when the optimal RA actions are achieved, it can be shown that

$$
\sum_{k} \mu_{k}=C=\frac{1}{\eta} \log _{2}\left(1+\frac{P}{N}\right),
$$

where $\eta=L / T_{s} W>0$. Otherwise we can reduce the value of $C$ and $P$ such that the objective function can be further 
maximized without any violation of the constraints in (27b)(27d). From (28), we have the following power consumption of $C$ :

$$
P=N\left(2^{\eta C}-1\right)
$$

and constraints (27b) and (27d) further imply that

$$
0 \leq C \leq \min \left(\sum_{k \in \mathscr{K}} Q_{k}, \widetilde{C}_{\max }\right)
$$

where $\widetilde{C}_{\max } \triangleq(1 / \eta) \log _{2}\left(1+P_{\max } / N\right)$.

Secondly, the resource allocation subproblem (27a)(27d) can be equivalently transformed into a single variable problem as follows:

$$
\begin{array}{ll}
\max _{C \in \mathbb{N}} & \widehat{M}(C) \triangleq g_{1}(C)-g_{2}(C) \\
\text { s.t. } & (30),
\end{array}
$$

where $g_{1}(C)$ is given by

$$
\begin{aligned}
g_{1}(C) \triangleq \max _{\left\{\mu_{k}\right\}} & \sum_{k \in \mathscr{K}} Q_{k} \mu_{k} \\
\text { s.t. } \quad & 0 \leq \mu_{k} \leq Q_{k}, \quad \mu_{k} \in \mathbb{N}, \quad \forall k \in \mathscr{K}, \\
& \sum_{k \in \mathscr{K}} \mu_{k}=C,
\end{aligned}
$$

and $g_{2}(C)$ is given by

$$
g_{2}(C) \triangleq \sum_{k=1}^{K} X_{k} P=\sum_{k=1}^{K} X_{k}\left(2^{\eta C}-1\right) N=\rho\left(2^{\eta C}-1\right) \text {, }
$$

with $\rho \triangleq N \sum_{k=1}^{K} X_{k}$.

Now let us focus on the problem (32a)-(32c) with any given $C$. Clearly, the maximum objective value of (32a)-(32c) can always be achieved by allocating link capacity $C$ to the services in the descending order of their backlogs, which is similar to the max-weight algorithm in [15]. Hence, we sort all the services in descending order of $Q_{k}$ and denote the ordered set by $\left\{k_{1}, k_{2}, \ldots, k_{K}\right\}$. For convenience, we define $f(m)=\sum_{n=0}^{m} Q_{k_{n}}(t)$ for $m=0,1, \ldots, K$, where $Q_{k_{0}}(t)=0$. One can see that $f(m)$ is an increasing function of $m$, and $0 \leq C \leq f(K)$ from (30). Therefore the optimal solutions to the problem (32a) $-(32 \mathrm{c})$ are given by

$$
\mu_{k_{n}}= \begin{cases}Q_{k_{n}}, & \text { if } 1 \leq n<m, \\ C-f(n-1), & \text { if } n=m, \\ 0, & \text { if } m<n \leq K\end{cases}
$$

where $m \in\{1, \ldots, K\}$ such that $C \in(f(m-1), f(m)]$ if $C>0$; otherwise $\mu_{k_{n}}=0$ for all $n$.

Next, let us focus on problem (31a)-(31b). Indeed, we have the following lemma.

Lemma 5. $\widehat{M}(C)$ is a unimodal function of $C$ over $[0, f(K)]$.
Proof. On the one hand, since $\Delta g_{1}(C)=g_{1}(C+1)-g_{1}(C)=$ $Q_{k_{m}}$ for $f(m-1) \leq C(t)<f(m), \forall m \in[1, K], \Delta g_{1}(C)$ is a monotonically nonincreasing function of $C$. On the other hand, since $\Delta g_{2}(C)=\rho\left(2^{\eta}-1\right) 2^{\eta C}, \Delta g_{2}(C)$ is a monotonically increasing function of $C$. Therefore $\Delta \widehat{M}(C)=\Delta g_{1}(C)-\Delta g_{2}$ $(C)$, which is a monotonically decreasing function of $C$. For any $C \in[1, f(K)-1], \Delta \widehat{M}(C-1)>\Delta \widehat{M}(C)$, which implies $\widehat{M}(C)>(1 / 2)(\widehat{M}(C-1)+\widehat{M}(C+1))$, so $\widehat{M}(C)$ is concave on $[0, f(K)]$. Based on [28], if $\widehat{M}(C)$ is concave, then $\widehat{M}(C)$ is unimodal.

Based on Lemma 5, since $\widehat{M}(C)$ is a unimodal function of $C$ over $\left[0, \min \left\{\widetilde{C}_{\max }, f(K)\right\}\right]$, the golden section search method [29] can be used to obtain the global optimal solution to the problem (31a)-(31b). However, this method requires the knowledge of all queue backlog information. When the center controller is not available, a distributed resource allocation scheme is highly desirable. Relying on the insights from Lemma 5, we propose a distributed resource allocation scheme, where each CS can communicate with all other CSs, and the network resources are allocated packet by packet.

The proposed packet loading resource allocation scheme is detailed in Algorithm 1. For each slot, each CS exchanges the backlog information with all other CSs and the order of the backlogs is obtained by all CSs (step 2). Then the packets of the services are fetched from the corresponding CS in descending order of their backlogs. When one CS fetches a new packet (step 5), $\widehat{M}(C)$ is calculated (step 6). This will be repeated until the optimal condition in step 7 is satisfied, which implies that $\widehat{M}(C-1)$ is the maximum or constraint (30) is violated, and thus step 8 should be performed since the last packet cannot be transmitted. When one CS empties its buffer, it should send the value information of $C$ to the next CS (step 12), and the next CS should continue the resource allocation. Here we remark that the optimal solutions can be achieved by the proposed scheme, which can be readily proved by Lemma 5 .

4.6. Distributed Dynamic AC and RA Algorithm. Based on the above three separate subproblems, we propose a distributed dynamic AC and RA algorithm as shown in Algorithm 2. All system parameters should be initialized before the trip begins. At each slot, each CS solves three subproblems in steps 4,5 , and 6 . At the end of each slot, the queue vector $\Theta(t+1)$ is updated according to (4), (7), and (9). This algorithm will be repeated when the train travels from the origin station to the destination station.

Remark 6 (utility-backlog tradeoff). Based on [15], the achieved utility differs from optimality by $\mathcal{O}(1 / V)$, in the sense that

$$
\phi_{1}^{*}-\liminf _{t \rightarrow \infty} \sum_{k \in \mathscr{K}} \phi_{k}\left(\bar{r}_{k}\right) \leq \mathcal{O}\left(\frac{1}{V}\right)
$$

where $\phi_{1}^{*}$ is the maximal utility for the problem (P1). It implies that the proposed algorithm can achieve a utility which is arbitrarily close to $\phi_{1}^{*}$ by increasing $V$. In addition, the actual 


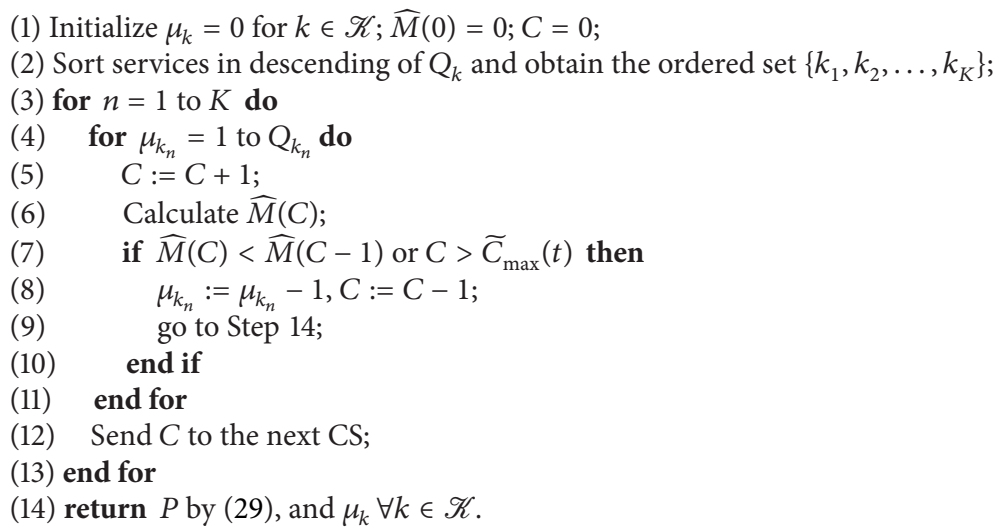

Algorithm 1: Packet loading resource allocation scheme for problem (27a) - (27d).

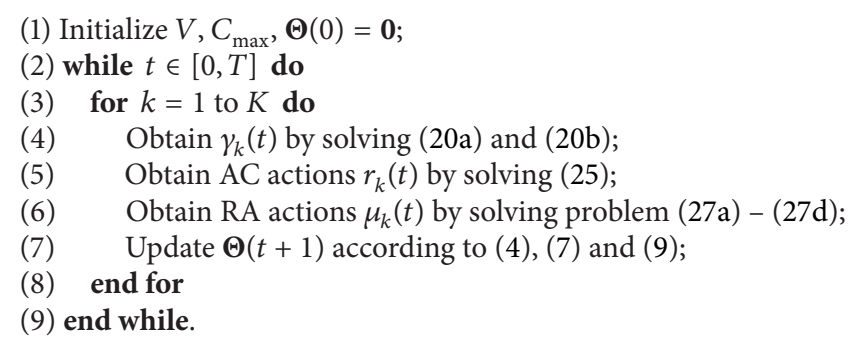

Algorithm 2: Distributed dynamic AC and RA algorithm for (P2).

queue backlog of each service grows linearly with $V$, which is given by

$$
\limsup _{t \rightarrow \infty} \bar{Q}_{k} \leq \frac{D}{\epsilon}+\mathcal{O}(V)
$$

where $\epsilon>0$ is a parameter and $D$ is defined in (14). Therefore, the above expressions (35) and (36) present a utility-backlog tradeoff of $[\mathcal{O}(1 / V), \mathcal{O}(V)]$.

Recalling the utility functions (21) and (23), we observe that they have the maximum right derivatives $\nu_{k}>0$ over the interval $0 \leq \gamma_{k}(t) \leq B_{k}$. Based on this observation, we obtain the boundedness property on the virtual queue $Z_{k}(t)$ in the following lemma.

Lemma 7. If the utility function $\phi_{k}\left(\gamma_{k}\right)$ has maximum right derivatives $v_{k}>0$, then the backlog of virtual queue $Z_{k}(t)$ satisfies

$$
0 \leq Z_{k}(t) \leq V v_{k}+B_{k}, \quad \forall t \in[0, T]
$$

provided that this inequality holds for $Z_{k}(0)$.

Proof. The proof of Lemma 7 is provided in Appendix C.
TABLE 1: Parameters in simulation.

\begin{tabular}{lcc}
\hline Parameter & Description & Value \\
\hline$P_{\mathrm{av}}$ & Average power constraint & $35 \mathrm{~W}$ \\
$B$ & System bandwidth & $5 \mathrm{MHz}$ \\
$L$ & Packet size & $240 \mathrm{bits}$ \\
$T_{s}$ & Slot duration & $1 \mathrm{~ms}$ \\
$\alpha$ & Pathloss exponent & 4 \\
$P_{\max }$ & Maximum transmit power & $45 \mathrm{~W}$ \\
$v$ & Constant moving speed & $100 \mathrm{~m} / \mathrm{s}$ \\
$R$ & Cell radius & $1.5 \mathrm{~km}$ \\
$d_{0}$ & Distance between BS and rail & $50 \mathrm{~m}$ \\
$K$ & Number of services & 6 \\
\hline
\end{tabular}

\section{Numerical Results and Discussions}

In this section, we implement the proposed distributed dynamic AC and RA algorithm using MATLAB and present simulation results to illustrate the performance of it. We use the piecewise linear utility functions (21) for all services and summarize the simulation parameters in Table 1 . The packet size $L$ is set to 240 bits according to $[12,30]$, and the slot duration $T_{s}$ is set to $1 \mathrm{~ms}$ according to [31]. A single simulation runs the proposed algorithm when the train moves through a cell (30,000 slots). 


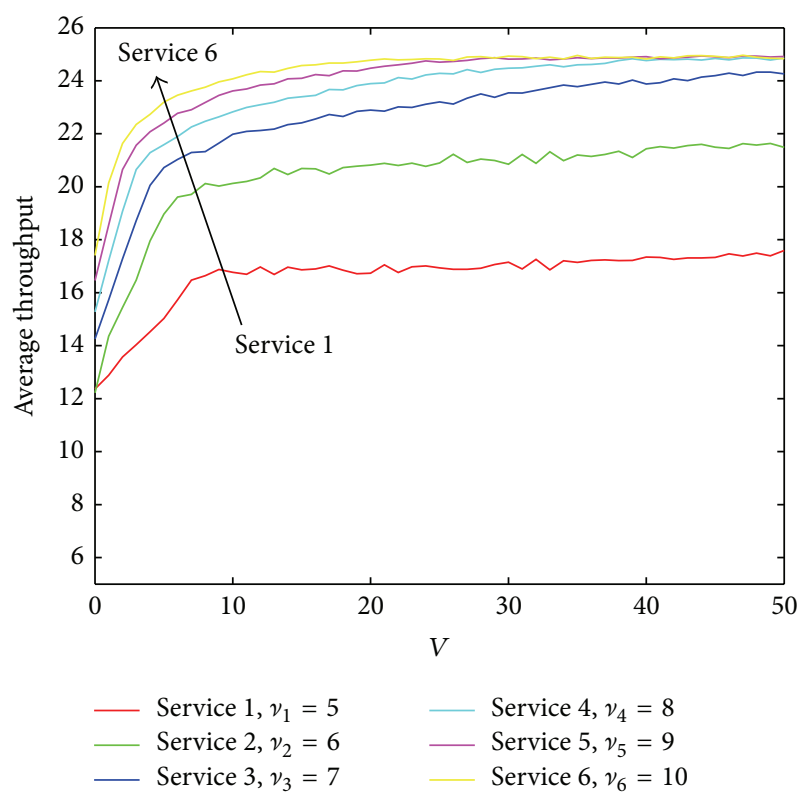

Figure 2: Average throughput with different $V$.

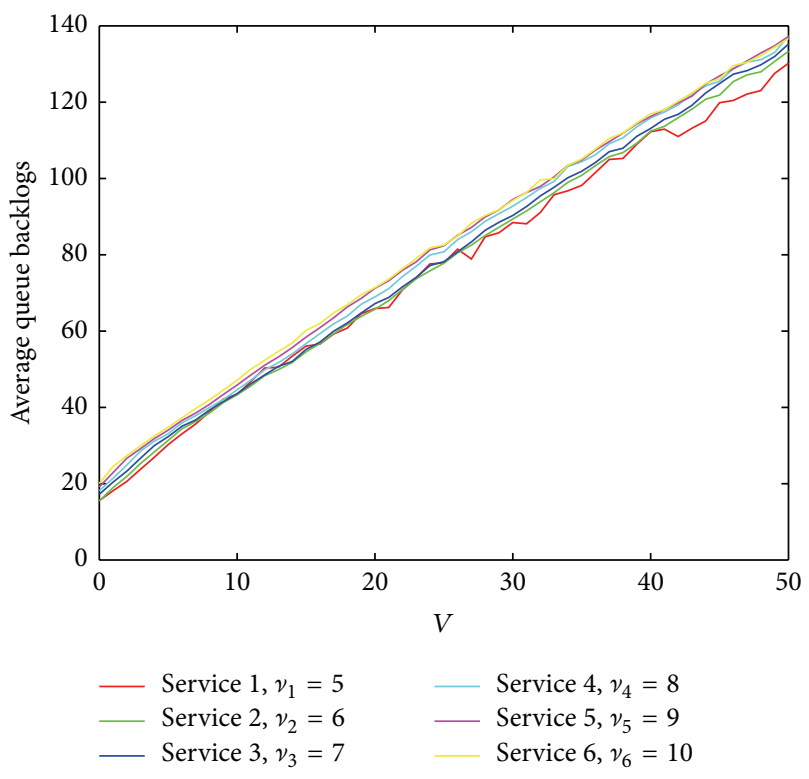

FIGURE 3: Average queue backlog with different $V$.

Figures 2 and 3 explore the throughput-backlog tradeoff with different $V$. In the simulations, we use the same parameters $\lambda_{k}=25$ and $x_{k}=1$ and different priorities for all services. As shown in Figure 2, the average throughput for each service increases as $V$ is increased and the service with high priority gets the large average throughput. Figure 3 presents that the average queue backlogs of all services are linearly increasing with $V$, which demonstrates the $\mathcal{O}(V)$ behavior in (36). Furthermore, the proposed algorithm can ensure that the average queue backlogs of the services with different priorities are almost the same.

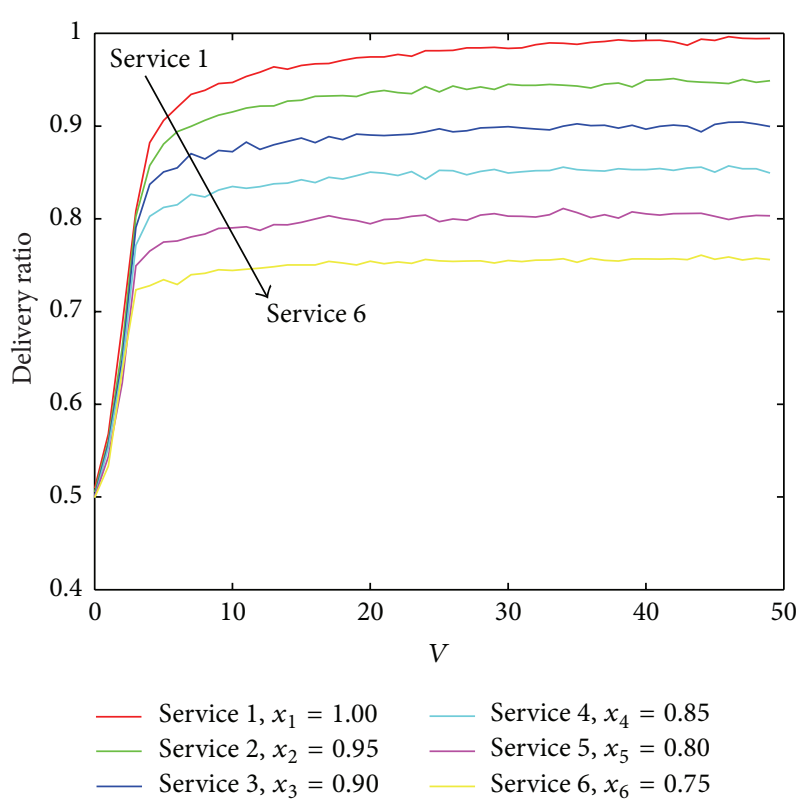

FIgURE 4: Delivery ratio with different $V$.

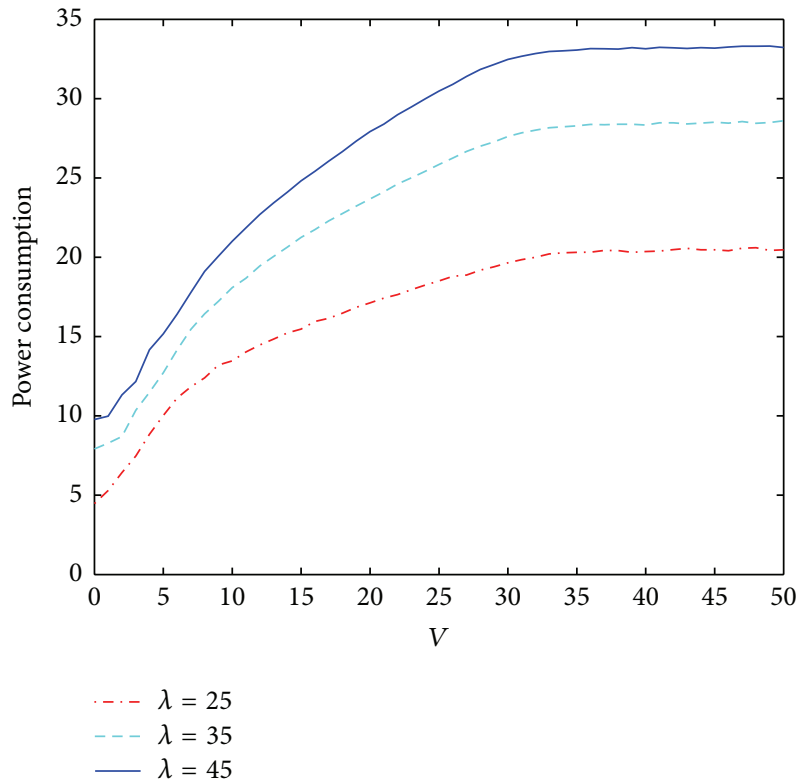

Figure 5: Power consumption with different $V$.

Figure 4 illustrates the achieved delivery ratios for the services with different maximum desired delivery ratios and same arrival rate $\lambda_{k}=25$ as well as priority $\nu_{k}=10$. It can be observed that the large $V$ will result in the improvement of the delivery ratio performance. This can be explained as follows: since a larger $V$ gives a higher priority on throughput, more packets will be admitted into the buffers, which causes the higher delivery ratio performance. In addition, the delivery ratio for each service is close to its maximum desired delivery ratio when $V$ is larger than 40, which implies that the 
proposed algorithm can archive different maximum desired delivery ratios when a large $V$ is chosen.

Figure 5 compares the average power consumption under different arrival rate conditions. In this simulation, we set the same parameters $v_{k}=10, x_{k}=1$, and $\lambda_{k}=\lambda$ for all services.From this figure, we can see that the average power consumption increases as $V$ is increased. This is exactly what happens. A larger $V$ results in more packets admitted into buffers, while transmitting these packets will cost more power. As for the same $V$, a larger $\lambda$ will cause more power consumption, since more packets will be admitted into buffers based on (26). In addition, the average power consumption can satisfy the average power constraint when the arrival rate is $\lambda_{k}=25$, which is reasonably set in the previ-ous simulations.

Figure 6 describes the backlog update processes of virtual queues $Z_{k}(t)$ for three types of services. In the simulation, we set the same parameters $\nu_{k}=10, x_{k}=1$, and $\lambda_{k}=35$ for all services and $V=100$. From the figure, we can see $0 \leq Z_{k}(t) \leq$ $V v_{k}+B_{k}$ for all services at all slots, which illustrates the boundedness property on queue backlogs in Lemma 7 .

\section{Conclusion}

In this paper, we formulate the joint admission control and resource allocation problem under average power constraint for multimedia services delivery in HSR wireless networks. With the help of virtual queues, the original stochastic optimization problem is transformed into a queue stability problem, which is decomposed into three separate subproblems by the drift-plus-penalty approach. It is worth noting that the optimal solution to the resource allocation subproblem can be obtained by the packet loading resource allocation scheme. Based on the stochastic optimization technique, the dynamic admission control and resource allocation algorithm is proposed, which is suitable for distributed implementation in HSR wireless networks. Furthermore, the performance of the proposed algorithm is analyzed theoretically and validated by numerical simulations under realistic conditions for HSR wireless networks. For future work, we will further investigate the effects of the nonmentioned parts in the communication system, such as frame error check blocks and adaptive channel equalizers.

\section{Appendices}

\section{A. Proof of Lemma 3}

Let $\phi_{1}^{*}$ and $\phi_{2}^{*}$ be the optimal utility of problems (P1) and (P2), respectively.

First, to prove $\phi_{1}^{*} \geq \phi_{2}^{*}$, let $\alpha_{2}^{*}(t)$ be an optimal solution achieving $\phi_{2}^{*}$ in problem (P2), which includes $\gamma_{k}^{*}(t), r_{k}^{*}(t)$, and $\mu_{k}^{*}(t)$ at slot $t$. Since $\phi(\cdot)$ is concave, based on Jensen's inequality, we have

$$
\sum_{k \in \mathscr{K}} \phi\left(\overline{\gamma_{k}^{*}}\right) \geq \sum_{k \in \mathscr{K}} \overline{\phi\left(\gamma_{k}^{*}\right)}=\phi_{2}^{*}
$$

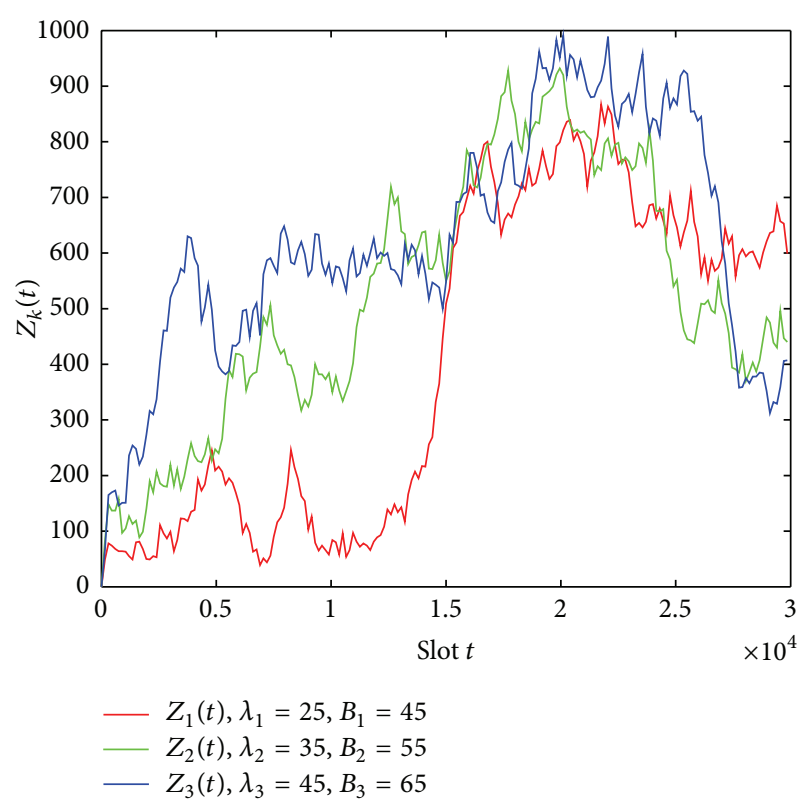

FIGURE 6: The backlog update processes of virtual queues $Z_{k}(t)$.

In addition, since the solution $\alpha_{2}^{*}(t)$ satisfies constraint (8b) and $\phi(\cdot)$ is nondecreasing, we further have

$$
\sum_{k \in \mathscr{K}} \phi\left(\overline{r_{k}^{*}}\right) \geq \sum_{k \in \mathscr{K}} \phi\left(\overline{\gamma_{k}^{*}}\right)
$$

Since the constraints in problem (P2) include all of the desired constraints of the original problem $(\mathrm{P} 1), \alpha_{2}^{*}(t)$ is a feasible solution for problem (P1) which gives a utility that is not larger than $\phi_{1}^{*}$. Thus we conclude that

$$
\phi_{1}^{*} \geq \sum_{k \in \mathscr{K}} \phi\left(\overline{r_{k}^{*}}\right) \geq \phi_{2}^{*}
$$

Next, to prove $\phi_{1}^{*} \leq \phi_{2}^{*}$, let $\alpha_{1}^{*}(t)$ be an optimal solution achieving $\phi_{1}^{*}$ for problem (P1), which includes $r_{k}^{*}(t)$ and $\mu_{k}^{*}(t)$ at slot $t$. Since $\alpha_{1}^{*}(t)$ satisfies constraints (6b)-(6f), it also satisfies constraints (8d) of the problem (P2). Further, for all $k \in \mathscr{K}$, we set $\gamma_{k}(t)=\overline{r_{k}^{*}}$ at all time $t$ which can satisfy constraints (8b) and (8c). Thus, such choice of $\gamma_{k}(t)$ together with the solution $\alpha_{1}^{*}(t)$ forms a feasible solution for the problem (P2). By definition, $\overline{\phi\left(\gamma_{k}\right)}=\lim _{t \rightarrow \infty}(1 / t) \sum_{\tau=0}^{t-1} \phi\left(\gamma_{k}(\tau)\right)=$ $\lim _{t \rightarrow \infty}(1 / t) \sum_{\tau=0}^{t-1} \phi\left(\overline{r_{k}^{*}}\right)=\phi\left(\overline{r_{k}^{*}}\right)$. Therefore, we get

$$
\phi_{2}^{*} \geq \sum_{k \in \mathscr{K}} \overline{\phi\left(\gamma_{k}\right)}=\sum_{k \in \mathscr{K}} \phi\left(\overline{r_{k}^{*}}\right)=\phi_{1}^{*}
$$

From the above analysis, we can conclude that $\phi_{1}^{*}=\phi_{2}^{*}$ based on (A.3) and (A.4) and that an optimal solution for the problem (P2) can be directly turned into an optimal solution for the problem (P1). 


\section{B. Proof of Lemma 4}

Recall the evolution equation (9) for the queue $X_{k}(t)$ and by squaring this equation, we obtain

$$
\begin{aligned}
& X_{k}(t+1)^{2}-X_{k}(t)^{2} \\
&=\left(\max \left[X_{k}(t)-P_{\mathrm{av}}, 0\right]+P(t)\right)^{2}-X_{k}(t)^{2} \\
& \leq\left[P(t)^{2}+P_{\mathrm{av}}^{2}\right]+2 X_{k}(t)\left[P(t)-P_{\mathrm{av}}\right]
\end{aligned}
$$

where in the final inequality we have used the following facts: $\left(\max \left[X_{k}(t)-P_{\mathrm{av}}, 0\right]\right)^{2} \leq\left(X_{k}(t)-P_{\mathrm{av}}\right)^{2}$ and $\max \left[X_{k}(t)-\right.$ $\left.P_{\mathrm{av}}, 0\right] \leq X_{k}(t)$.

Similarly, it can be shown for $k \in \mathscr{K}$ that

$$
\begin{aligned}
Z_{k}(t+1)^{2}-Z_{k}(t)^{2} & \\
& \leq\left[\gamma_{k}(t)^{2}+r_{k}(t)^{2}\right]+2 Z_{k}(t)\left[\gamma_{k}(t)-r_{k}(t)\right] \\
Q_{k}(t+1)^{2}-Q_{k}(t)^{2} & \\
& \leq\left[r_{k}(t)^{2}+\mu_{k}(t)^{2}\right]+2 Q_{k}(t)\left[r_{k}(t)-\mu_{k}(t)\right] .
\end{aligned}
$$

Based on (12) and (B.1a)-(B.2), we have

$$
\begin{aligned}
& \Delta(\Theta(t))=\mathbb{E}\left[\frac { 1 } { 2 } \sum _ { k \in \mathscr { K } } \left[X_{k}(t+1)^{2}-X_{k}(t)^{2}\right.\right. \\
& +Q_{k}(t+1)^{2}-Q_{k}(t)^{2} \\
& \left.\left.+Z_{k}(t+1)^{2}-Z_{k}(t)^{2}\right] \mid \Theta(t)\right] \\
& \leq \mathbb{E}\left[\frac { 1 } { 2 } \sum _ { k \in \mathscr { K } } \left[P(t)^{2}+P_{\mathrm{av}}^{2}+\gamma_{k}(t)^{2}\right.\right. \\
& \left.\left.+r_{k}(t)^{2}+r_{k}(t)^{2}+\mu_{k}(t)^{2}\right] \mid \Theta(t)\right] \\
& +\mathbb{E}\left[\sum _ { k \in \mathscr { K } } \left[X_{k}(t)\left[P(t)-P_{\mathrm{av}}\right]\right.\right. \\
& +Z_{k}(t)\left[\gamma_{k}(t)-r_{k}(t)\right] \\
& \left.\left.+Q_{k}(t)\left[r_{k}(t)-\mu_{k}(t)\right]\right] \mid \Theta(t)\right] \\
& \leq D+\mathbb{E}[G(t) \mid \boldsymbol{\Theta}(t)],
\end{aligned}
$$

where $G(t)$ is defined by (15) and the last inequality can be obtained as follows. For any slot $t$, any possible packet arrival vector $\mathbf{A}(t)$, and any possible $P(t)$ as well as RA actions that can be taken, we have

$$
\begin{gathered}
\mathbb{E}\left[\frac{1}{2} \sum_{k \in \mathscr{K}}\left[P(t)^{2}+P_{\mathrm{av}}^{2}+\gamma_{k}(t)^{2}+2 r_{k}(t)^{2}+\mu_{k}(t)^{2}\right] \mid \Theta(t)\right] \\
\leq \mathbb{E}\left[\frac{1}{2} \sum_{k \in \mathscr{K}}\left[P_{\max }^{2}+P_{\mathrm{av}}^{2}+3 B_{k}^{2}+C_{\max }^{2}\right] \mid \Theta(t)\right] \\
\quad=\frac{1}{2} \sum_{k \in \mathscr{K}}\left[P_{\max }^{2}+P_{\mathrm{av}}^{2}+3 B_{k}^{2}+C_{\max }^{2}\right]=D
\end{gathered}
$$

where the inequality holds based on $P(t) \leq P_{\max }, r_{k}(t) \leq B_{k}$, and $\mu_{k}(t) \leq C_{\max }$ as well as (8c), and the equality holds since the constant in the square bracket is independent of queue vector $\Theta(t)$ at slot $t$.

\section{Proof of Lemma 7}

We prove this lemma by induction. Assume that $Z_{k}(t) \leq$ $V v_{k}+B_{k}$ for slot $t$ (it holds by assumption at slot $t=0$ ); then we prove it also holds for slot $t+1$. Firstly, we consider the case $Z_{k}(t) \leq V v_{k}$. From the queue update equation (7), we can see that this queue can increase by at most $B_{k}$ at each slot, and thus we have $Z_{k}(t+1) \leq V v_{k}+B_{k}$, proving the result for this case.

Secondly, we consider the case $V v_{k}<Z_{k}(t) \leq V v_{k}+B_{k}$. For each slot $t, \mathrm{CS}_{k}$ decides $\gamma_{k}(t)$ to maximize the following expression:

$$
V \phi\left(\gamma_{k}(t)\right)-Z_{k}(t) \gamma_{k}(t)
$$

Based on the property of the maximum derivative, for any $\gamma_{k}(t) \geq 0$, we obtain

$$
\begin{aligned}
V \phi & \left(\gamma_{k}(t)\right)-Z_{k}(t) \gamma_{k}(t) \\
& \leq V \phi(0)+V v_{k} \gamma_{k}(t)-Z_{k}(t) \gamma_{k}(t) \\
& =V \phi(0)+\gamma_{k}(t)\left[V v_{k}-Z_{k}(t)\right] \\
& \leq V \phi(0),
\end{aligned}
$$

where the equality holds if and only if $\gamma_{k}(t)=0$. Then the algorithm will choose $\gamma_{k}(t)=0$ to maximize expression (C.1), and we can obtain

$$
Z_{k}(t+1)=\max \left[Z_{k}(t)-r_{k}(t), 0\right] \leq Z_{k}(t) \leq V v_{k}+B_{k}
$$

Thus, $Z_{k}(t+1) \leq V v_{k}+B_{k}$ is satisfied for these two cases, which completes the proof.

\section{Conflict of Interests}

The authors declare that there is no conflict of interests regarding the publication of this paper. 


\section{Acknowledgments}

This work is supported by the Fundamental Research Funds for the Central Universities (Grant no. 2014YJS026), the Key Projects of State Key Lab of Rail Traffic Control and Safety (no. RCS2012ZZ004 and no. RCS2010ZT011), the Opening Project of The State Key Laboratory of Integrated Services Networks, Xidian University (Grant no. ISN14-09), and the Key Grant Project of Chinese Ministry of Education (no. 313006).

\section{References}

[1] G. Barbu, "E-Train-broadband communication with moving trains," Tech. Rep., Technology State of the Art, June 2010.

[2] L. Tian, J. Li, Y. Huang, J. Shi, and J. Zhou, "Seamless duallink handover scheme in broadband wireless communication systems for high-speed rail," IEEE Journal on Selected Areas in Communications, vol. 30, no. 4, pp. 708-718, 2012.

[3] J. Wang, H. Zhu, and N. J. Gomes, "Distributed antenna systems for mobile communications in high speed trains," IEEE Journal on Selected Areas in Communications, vol. 30, no. 4, pp. 675-683, 2012.

[4] Y. S. Zhao, X. Li, Y. Li, and H. Ji, "Resource allocation for highspeed railway downlink MIMO-OFDM system using quantumbehaved particle swarm optimization," in Proceedings of the IEEE International Conference on Communications, pp. 936940, 2013

[5] W. Luo, X. Fang, M. Cheng, and Y. Zhao, "Efficient multiplegroup multiple-antenna (MGMA) scheme for high-speed railway viaducts," IEEE Transactions on Vehicular Technology, vol. 62, no. 6, pp. 2558-2569, 2013.

[6] L. Georgiadis, M. J. Neely, and L. Tassiulas, "Resource allocation and cross-layer control in wireless networks," Foundations and Trends in Networking, vol. 1, no. 1, pp. 1-144, 2006.

[7] K. T. Phan, T. Le-Ngoc, M. van ser Schaar, and F. Fu, "Optimal scheduling over time-varying channels with traffic admission control: structural results and online learning algorithms," IEEE Transactions on Wireless Communications, vol. 12, no. 9, pp. 4434-4444, 2013.

[8] Y. Q. Dong, P. Y. Fan, and K. B. Letaief, "High speed railway wireless communications: efficiency v.s. fairness," IEEE Transactions on Vehicular Technology, vol. 63, no. 2, pp. 925-930, 2013.

[9] D. Pareit, E. V. de Velde, D. Naudts, and J. Bergs, "A novel network architecture for train-to-wayside communication with quality of service over heterogeneous wireless networks," EURASIP Journal on Wireless Communications and Networking, vol. 2012, article 114, 2012.

[10] O. B. Karimi, J. Liu, and C. Wang, "Seamless wireless connectivity for multimedia services in high speed trains," IEEE Journal on Selected Areas in Communications, vol. 30, no. 4, pp. 729-739, 2012.

[11] Y. S. Zhao, X. Li, Z. X. Liang, Y. Li, and H. Ji, "Multidimensional resource allocation strategy for high-speed railway MIMOOFDM system," in Proceedings of the IEEE Global Communications Conference (Globecom '12), pp. 1653-1657, 2012.

[12] H. Liang and W. Zhuang, "Efficient on-demand data service delivery to high-speed trains in cellular/infostation integrated networks," IEEE Journal on Selected Areas in Communications, vol. 30, no. 4, pp. 780-791, 2012.
[13] S. F. Xu, G. Zhu, C. Shen, and Y. Lei, "Delay-aware fair scheduling in relay-assisted high-speed railway networks," in Proceedings of the International Conference on Communications and Networking in China, pp. 17-21, 2013.

[14] Y. S. Zhao, X. Li, and H. Ji, "Radio admission control scheme for high-speed railway communication with MIMO antennas," in Proceedings of the IEEE International Conference on Communications, pp. 5005-5009, 2012.

[15] M. J. Neely, Stochastic Network Optimization with Application to Communication and Queueing Systems, Morgan \& Claypool, 2010.

[16] S. Meyn and R. L. Tweedie, Markov Chains and Stochastic Stability, Cambridge University Press, Cambridge, UK, 2nd edition, 2009.

[17] Y. Cui, V. K. N. Lau, R. Wang, H. Huang, and S. Zhang, "A survey on delay-aware resource sontrol for wireless systems-large deviation theory, stochastic Lyapunov drift, and distributed stochastic learning," IEEE Transactions on Information Theory, vol. 58, no. 3, pp. 1677-1701, 2012.

[18] L. Tassiulas and A. Ephremides, "Stability properties of constrained queueing systems and scheduling policies for maximum throughput in multihop radio networks," IEEE Transacations on Automatic Control, vol. 37, no. 12, pp. 1936-1948, 1992.

[19] B. L. Niu, V. W. S. Wong, and R. Schober, "Downlink scheduling with transmission strategy selection for multi-cell MIMO systems," IEEE Transactions on Wireless Communications, vol. 12, no. 2, pp. 736-747, 2013.

[20] Z. Zhou, F. M. Liu, H. Jin, B. Li, B. C. Li, and H. B. Jiang, "On arbitrating the power-performance tradeoff in SaaS clouds," in Proceedings of the IEEE INFOCOM, pp. 872-880, 2013.

[21] S. Spagna, M. Liebsch, and R. Baldessari, "Design principles of an operator-owned highly distributed content delivery network," IEEE Communications Magazine, vol. 51, no. 4, pp. 132140,2013

[22] L. Liu, C. Tao, J. Qiu et al., "Position-based modeling for wireless channel on high-speed railway under a viaduct at $2.35 \mathrm{GHz}$," IEEE Journal on Selected Areas in Communications, vol. 30, no. 4, pp. 834-845, 2012.

[23] R. He, Z. Zhong, B. Ai, and J. Ding, "An empirical path loss model and fading analysis for high-speed railway viaduct scenarios," IEEE Antennas and Wireless Propagation Letters, vol. 10, pp. 808-812, 2011.

[24] Y. Dong, Q. Wang, P. Fan, and K. B. Letaief, "The deterministic time-linearity of service provided by fading channels," IEEE Transactions on Wireless Communications, vol. 11, no. 5, pp. 1666-1675, 2012.

[25] C. E. Shannon, "A mathematical theory of communication," ACM SIGMOBILE Mobile Computing and Communications Review, vol. 5, no. 1, pp. 3-55, 2001.

[26] D. Bethanabhotla, G. Caire, and M. J. Neely, "Utility optimal scheduling and admission control for adaptive video streaming in small cell networks," in Proceedings of the IEEE International Symposium on Information Theory (ISIT '13), 2013.

[27] G. L. Nemhauser and L. A. Wolsey, Integer and Combinatorial Optimization, John Wiley \& Sons, New York, NY, USA, 1988.

[28] E. M. J. Bertin and R. Theodorescu, "Some characterizations of discrete unimodality," Statistics and Probability Letters, vol. 2, no. 1, pp. 23-30, 1984.

[29] S. L. S. Jacoby, J. S. Kowalik, and J. T. Pizzo, Iterative Methods for Nonlinear Optimization Problems, Prentice-Hall, 1972. 
[30] D. H. Ho and S. Valaee, "Information raining and optimal linklayer design for mobile hotspots," IEEE Transactions on Mobile Computing, vol. 4, no. 3, pp. 271-284, 2005.

[31] R. Atat, E. Yaacoub, M. Alouini, and A. Dayya, "Heterogeneous LTE/802. 1la mobile relays for data rate enhancement and energy-efficiency in high speed trains," in Proceedings of the IEEE Global Communications Conference (Globecom '12), pp. 421-425, 2012. 


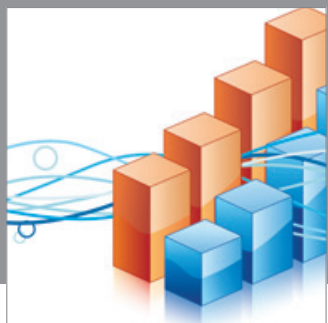

Advances in

Operations Research

mansans

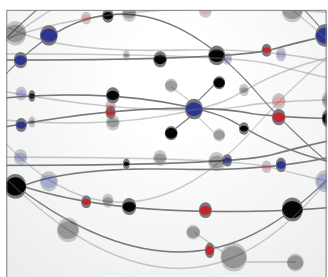

The Scientific World Journal
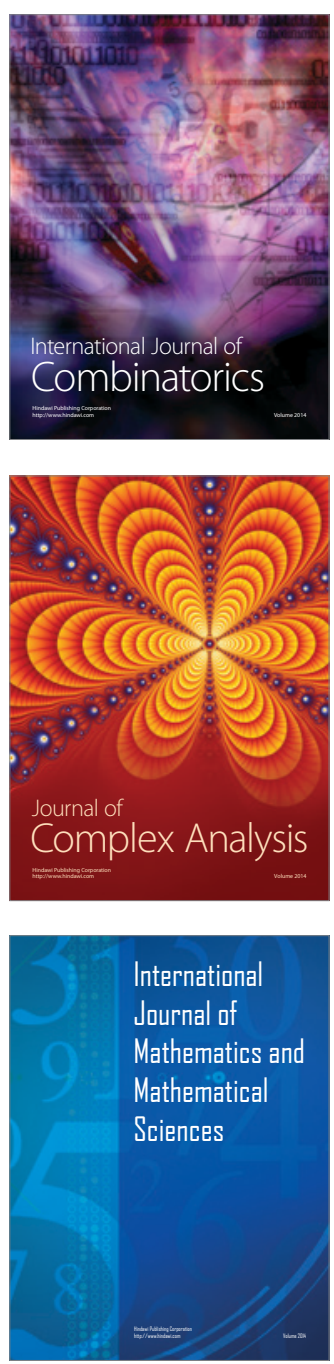
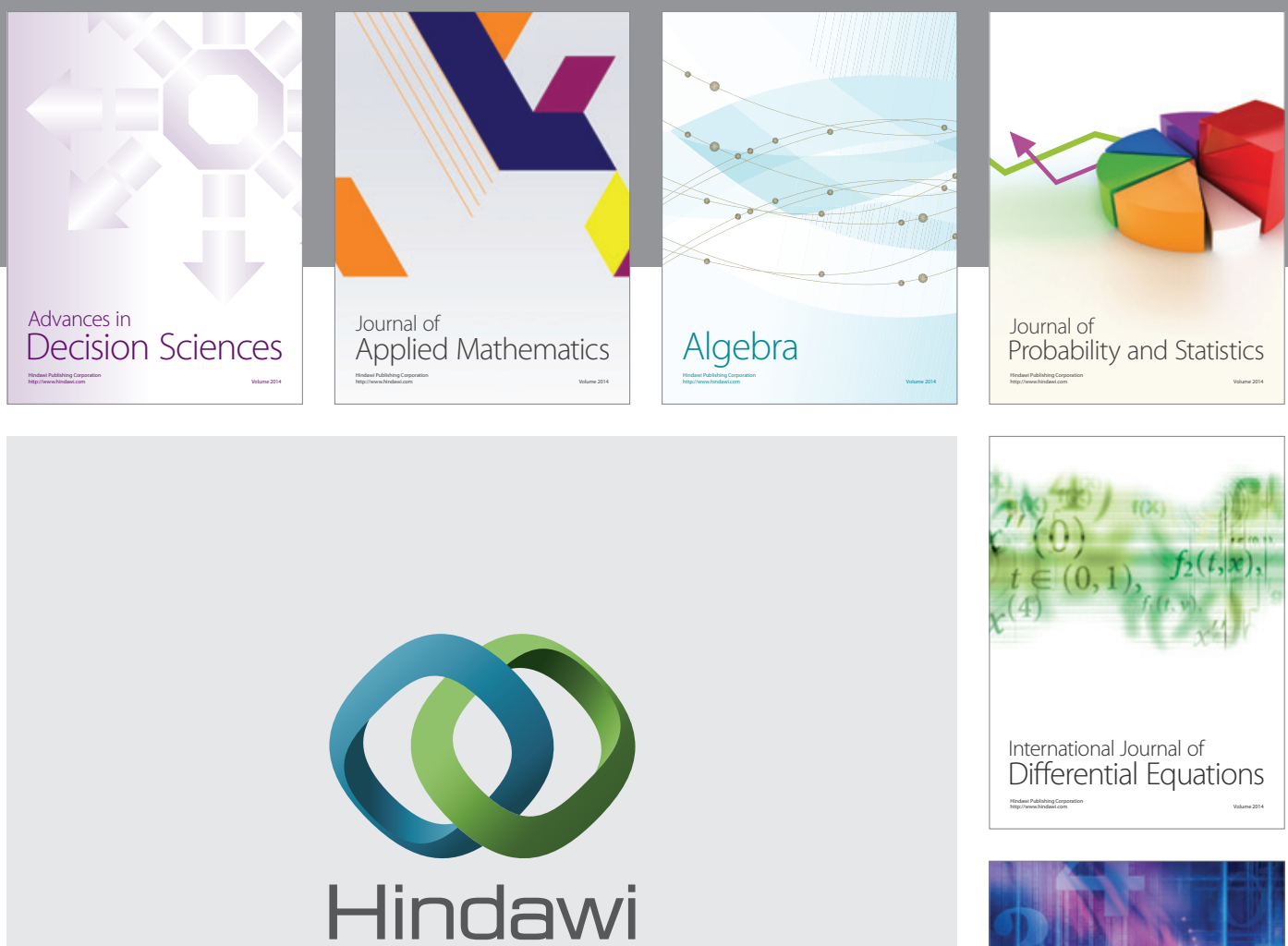

Submit your manuscripts at http://www.hindawi.com
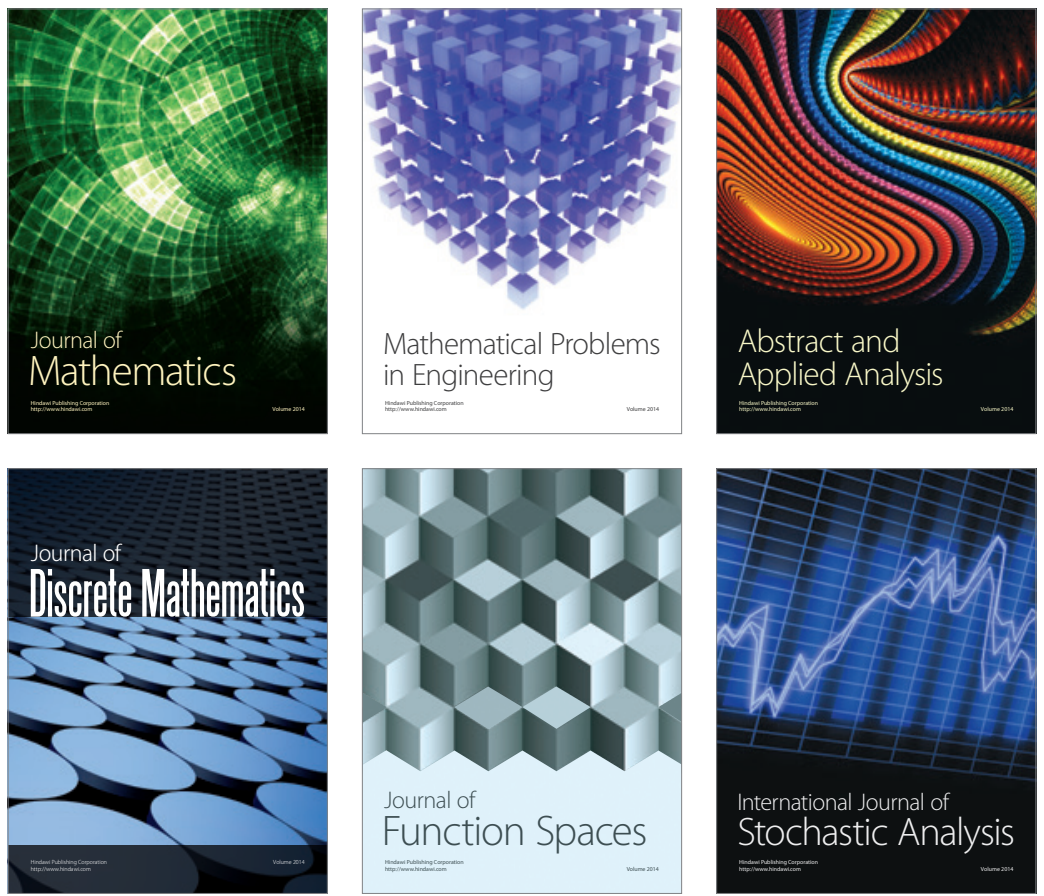

Journal of

Function Spaces

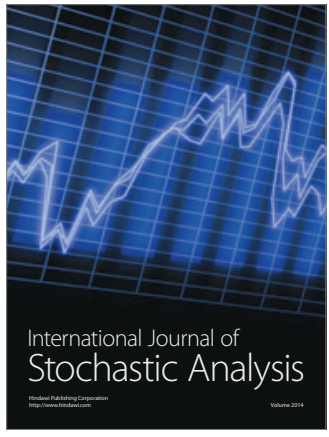

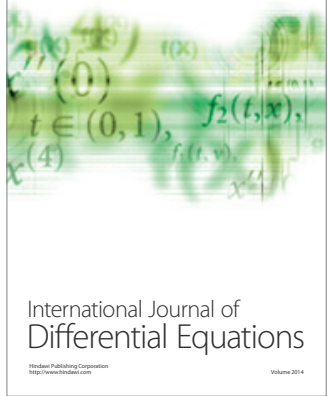
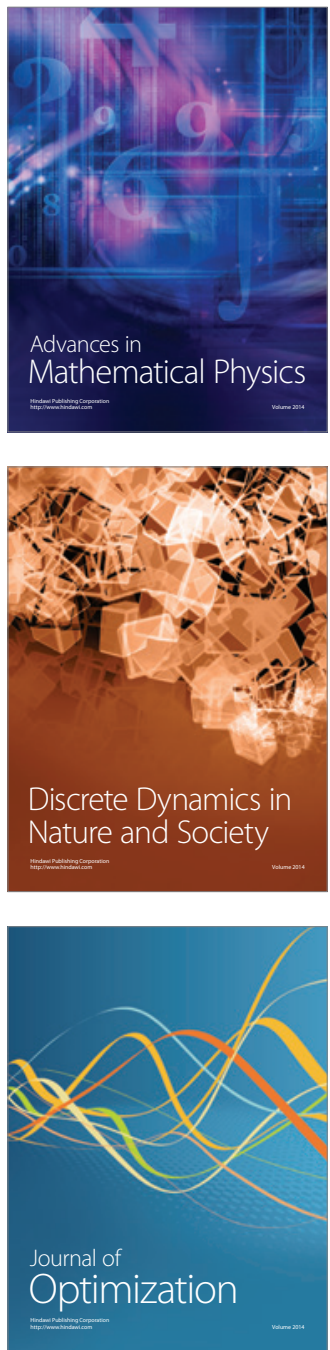